\title{
HACIA UNA REVISIÓN OBJETIVA DE LA TRADUCCIÓN
}

\author{
Ahmed Kamal Zaghloul \\ (Prof. visitante, Univ. Rey Saúd, Arabia Saudí, \\ Prof. titular, Uinv. Al-Azhar, Egipto)
}




\title{
HACIA UNA REVISIÓN OBJETIVA DE LA TRADUCCIÓN
}

\author{
Ahmed Kamal Zaghloul \\ (Prof. visitante, Univ. Rey Saúd, Arabia Saudí, \\ Prof. titular, Uinv. Al-Azhar, Egipto) \\ akelsayed@ksu.edu.sa
}

الملخص

\section{نحو مراجعة منهجية للترجمة}

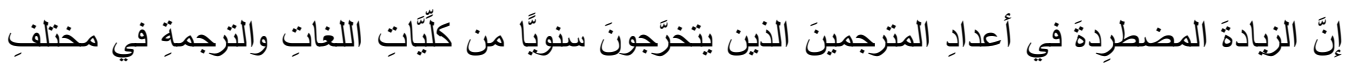

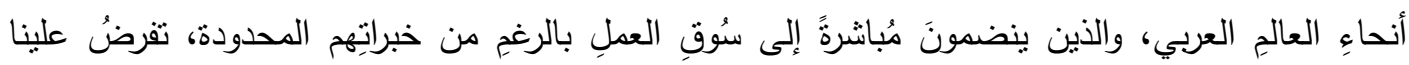

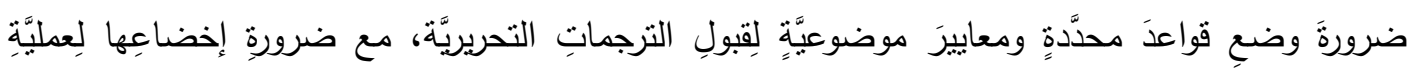

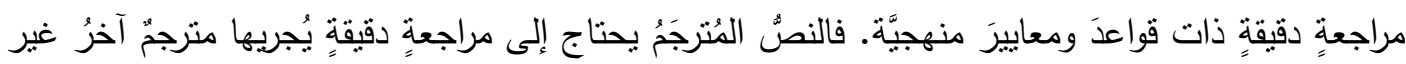

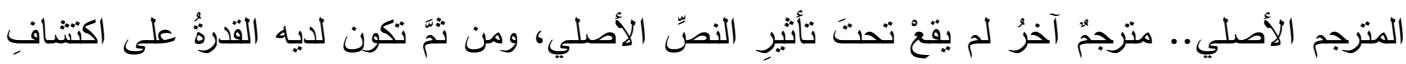

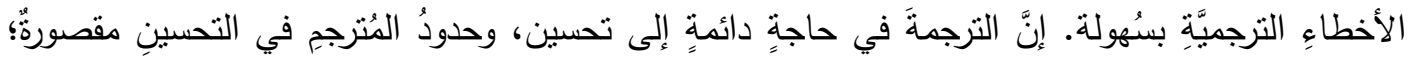

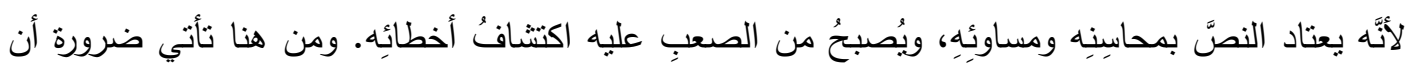

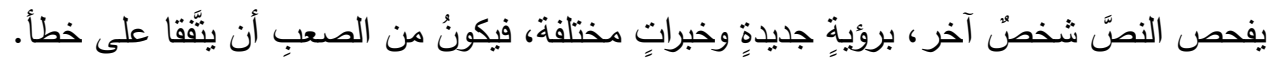

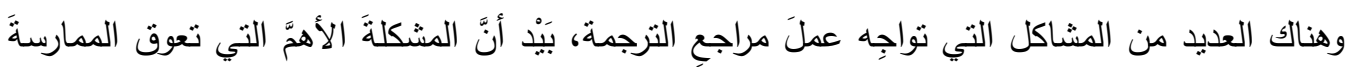

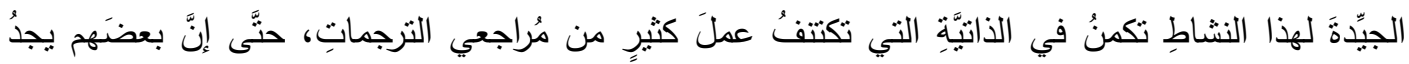

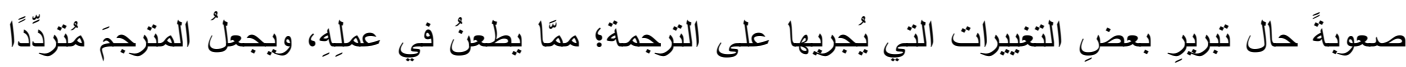

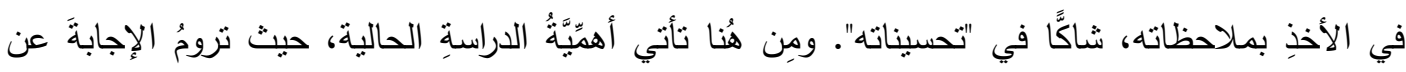

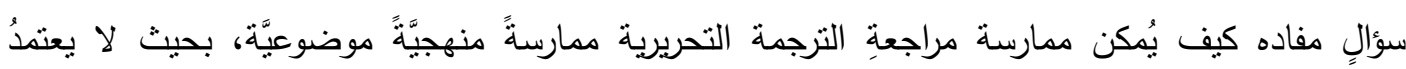

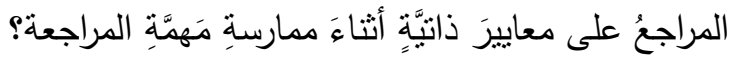

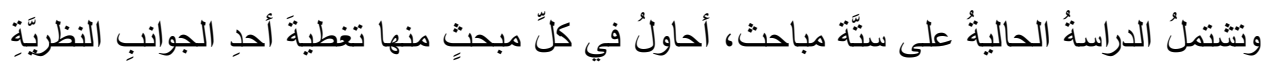

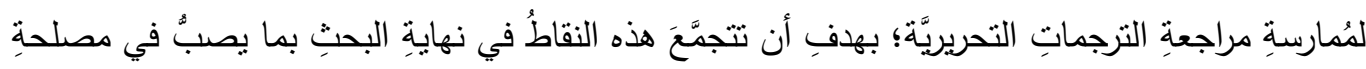

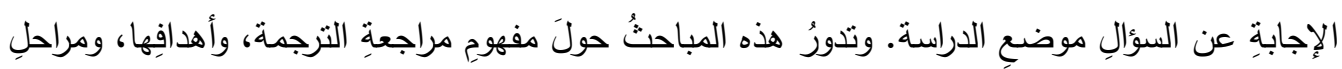

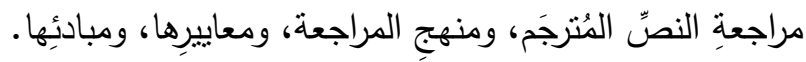




\title{
Towards an Objective Revision of the Translation
}

\begin{abstract}
The translated text needs to be reviewed by a person other than the translator, namely another translator who has not fallen under the influence of the original text, and, therefore, has the ability to discover translation errors. Translation requires constant improvement, given that the translator's ability to improve the arrival text could be limited because he gets used to it, with its advantages and disadvantages, and it becomes difficult to discover errors. There are several problems that face the work of the reviewer, although the probably most serious problem that hinders the good practice of this activity is the subjectivity with which many reviewers value and introduce changes to the arrival text, to the point where some reviewers find it difficult to justify their own changes, and that is why many translators sometimes hesitate to apply the changes and improvements of the reviewer. This study aims to answer the question of how to review the translation in an objective and methodological way, so that the revision is not based on personal values and subjective criteria.
\end{abstract}




\section{Introducción}

El constante aumento del número de traductores que se gradúan anualmente de las facultades de lenguas y traducción en el mundo árabe, y que se unen inmediatamente al mercado laboral de la traducción a pesar de su experiencia relativamente limitada, hace patente la necesidad de establecer normas específicas y criterios objetivos para aceptar las traducciones, además de someterlas a un proceso de revisión preciso y metodológico. Es ahí donde reside la importancia de la revisión en conseguir la calidad del texto traducido, ya que el traductor que sabe que su versión va a ser sometida a una revisión, presta mayor atención a la traducción. Asimismo, el texto traducido necesita ser revisado por una persona distinta del traductor, es decir, otro traductor que no haya caído bajo la influencia del texto original (TO), y, por lo tanto, tenga la capacidad de descubrir los errores de traducción.

La revisión de la traducción no es solamente importante, sino también necesaria en cualquier proyecto de traducción, ya sea realizado por una institución de traducción o por traductores autónomos. La traducción requiere constante mejora, dado que la capacidad del traductor a mejorar el texto de llegada (TL) podría ser limitada porque se acostumbra a él, con sus ventajas y desventajas, y se le vuelve difícil descubrir errores. De ahí surge la necesidad de examinar el texto traducido por parte de un tercero, que tenga una nueva visión y distintas experiencias. De esta manera, sería difícil que los dos, el traductor y el revisor, coincidieran en cometer los mismos errores.

Por otro lado, hay varios problemas que enfrentan la labor del revisor, aunque el problema probablemente más grave que obstaculiza la buena práctica de esta actividad consiste en la subjetividad con la cual muchos revisores valoran e introducen cambios al TL, hasta el punto de que a algunos les resulta difícil justificar sus propios cambios, razón por la cual muchos traductores vacilan a veces en aplicar los cambios y mejoras del revisor. De ahí la importancia del presente estudio, puesto que tiene por objetivo contestar a la pregunta de cómo se puede practicar la revisión de la traducción de una forma objetiva y metodológica, de modo que no se basa el revisor en valores personales y criterios subjetivos.

Para contestar a esta pregunta vamos a dividir este estudio en seis apartados, intentando en cada uno de ellos discutir uno de los aspectos teóricos de la actividad de la revisión. Dichos apartados giran en torno del concepto, objetivos, fases, método, parámetros y principios de la revisión de traducción. 


\section{Concepto de la revisión}

Sería conveniente comenzar este apartado por delimitar lo que se entiende por la palabra revisión en su sentido más amplio. El Diccionario de la Real Academia Española (DRAE) define la revisión como la "acción de revisar" (entendiéndose por revisar "someter una cosa a nuevo examen para corregirla, enmendarla o repararla") ${ }^{1}$. Es una definición aplicable a cualquier tipo de revisión; si la aplicamos al área de traducción, su significado sería corregir o enmendar el TL. Otra definición parecida a la del DRAE es la que expone Íñigo Valverde: "Se llama revisar a la acción de examinar algo, estudiarlo con el fin de comprobarlo y, si procede, modificarlo o enmendarlo"2 ${ }^{2}$. Ambas definiciones se enmarcan dentro del sentido general de la revisión, razón por la cual sería necesario averiguar otras definiciones que permiten explicar el sentido de la revisión de la traducción con mayor precisión.

La definición que figura en el Manual de revisión, publicado en 2010 por el Departamento de Español de la Dirección General de Traducción (DGT) de la Comisión Europea, es menos amplia que las dos anteriores, pues entiende la revisión como "comparación de una traducción con su original a fin de señalar o corregir posibles deficiencias, tanto en su contenido como en su presentación formal"3. Aunque esta definición sigue careciendo de la precisión debida, se distingue por exigir la consulta del TO para comprobar que el texto final es equivalente, es decir, el revisor no debería limitarse a leer únicamente el TL.

En el artículo titulado "Four Eyes are Better than Two", Peter Arthern trata de poner una definición clara y sistemática de la revisión de traducción, que es la siguiente:

The revision of a translation is a procedure by which it is examined and reviewed by a person or persons other than the translator, with or without consulting the latter, in order to ensure that it is an accurate and faithful rendering of the meaning of the original text into the language of the translation, in a style equivalent to that of the original ${ }^{4}$.

\footnotetext{
${ }^{1}$ Real Academia Española (ed.), Diccionario de la Lengua Española, Madrid, Espasa Calpe, 2014, $23^{a}$ ed., s. v. "revisión".

2 Í. Valverde, "Algunas consideraciones sobre la revisión", Puntoycoma 117 (2010), p. 34.

${ }^{3}$ Comisión Europea, Manual de revisión, Bruselas y Luxemburgo, Comisión Europea, 2010, p. 6.

${ }^{4}$ P. Arthern, "Four eyes are better than two", Translating and the Computer 8: A Profession on the Move, C. Picken (ed.), London, Aslib, 1987, p. 14. «La revisión de una traducción es un procedimiento mediante el cual el texto de llegada es examinado y revisado por una o varias personas distintas del traductor, con o sin consultar a este último, a fin de garantizar que sea una transmisión precisa y fiel del contenido del texto original en el idioma de la traducción, y en un estilo equivalente al del texto original» Traducción del autor (T. del A.).
} 
Según esta definición la revisión debe ser realizada por un tercero, es decir, la comprobación que hace el traductor de su propia traducción no es una revisión propiamente dicha. La definición de Arthern pone de manifiesto, asimismo, que el revisor puede examinar el TL con o sin consultar al traductor.

Una de las definiciones más claras y ajustadas de la revisión de la traducción es la que proponen Horguelin y Brunette en la tercera edición de su libro Pratique de la revisión, publicada en 1998. Según esta definición, la revisión de la traducción es:

Una etapa de la operación de traducción que consiste en realizar un examen global y atento de un texto traducido y de su original para, en su caso, restablecer la adecuación de la traducción a los criterios metodológicos y teóricos, lingüísticos, textuales y situacionales (en la nueva situación de enunciación) determinados previamente $^{5}$.

El uso de la palabra "etapa" en la definición anterior hace de la revisión una parte integrante del proceso de traducción. Parece que los autores quieren indicar implícitamente que la traducción no estaría completa sin una revisión. De esta manera, se puede distinguir entre la revisión de la traducción, por un lado, y la evaluación y la crítica de la traducción, por otro. Estas dos últimas prácticas trabajan, a diferencia de la revisión, sobre un producto acabado. La importancia de esta definición radica en su insistencia en que el revisor no debe cumplir su tarea partiendo de un enfoque meramente subjetivo, más bien debe seguir ciertos criterios objetivos determinados a priori.

La revisión de la traducción es definida en la norma europea de calidad para servicios de traducción EN-15038 como un "examen de una traducción respecto a su adecuación a la finalidad prevista, cotejo de los textos de origen y de destino, y recomendación de las correcciones pertinentes"6. Esta definición destaca la importancia de la finalidad del texto, es decir, no busca la equivalencia absoluta entre el TO y el TL sin tener en consideración los nuevos destinatarios, que podrían ser diferentes a los del TO.

Una de las definiciones de la revisión de la traducción más importante es, a nuestro parecer, la de Parra Galiano, quien la define de la manera siguiente:

Una lectura atenta de un texto de llegada (TL), considerado como producto semiacabado o borrador de traducción, que realiza la misma persona que ha traducido el texto (traductor) o un tercero (revisor), generalmente, comparándolo

\footnotetext{
5 Horguelin y Brunette, citado por S. Parra Galiano, "La revisión de traducciones en la traductología: aproximación a la práctica de la revisión en el ámbito profesional mediante el estudio de casos y propuestas de investigación", Universidad de Granada, 2005, p. 17.

${ }^{6}$ AENOR, Norma Española UNE-EN 15038. Servicios de traducción. Requisitos para la prestación del servicio, Madrid, AENOR, 2006, p. 7.
} 
con el texto de origen (TO) y utilizando para ello una serie de criterios establecidos a priori, con finalidad de comprobar si se han cumplido las especificaciones del encargo de traducción y realizar las oportunas correcciones y mejoras en el TL, antes de proceder a su entrega al cliente ${ }^{7}$.

A nuestro juicio, lo más importante en esta definición es que considera el texto traducido como inacabado hasta que sea revisado. Por ende, y según la última frase de la definición, el revisor no recomienda, sino realiza las correcciones y mejoras que cree necesarias.

Así, cada uno de los especialistas anteriores ha pretendido, a través de la definición que sugiere, delimitar lo más que pueda de los elementos constitutivos del concepto de la revisión de traducción, de acuerdo con la perspectiva que adopta hacia dicho concepto. De ahí, algunos creen que es imprescindible comparar el TL con el TO; otros exigen criterios fijos y objetivos determinados previamente para llevar a cabo la revisión; y otros creen que la revisión debe ser realizada por un tercero, etc.

Expuestas ya todas estas definiciones, sugiero a continuación nuestra propia definición de la revisión de traducción, partiendo de lo que considero características distinguibles e imprescindibles de la buena práctica de la revisión. La revisión de la traducción es, a nuestro juicio, una fase inherente al proceso de traducción, realizada por un segundo traductor, y sin la cual el proceso de traducción no se considera completo. En dicha fase, el revisor, partiendo de criterios y consideraciones previas y de una comparación del texto traducido con el original, verifica la exactitud de transmitir la totalidad del mensaje que contiene el TO, centrándose en las especificaciones explícitas e implícitas del encargo de traducción, con la finalidad de corregir y mejorar la traducción.

\section{Objetivos de la revisión}

Establecer metas y objetivos de la revisión de la traducción es, sin duda, una base fundamental para delimitar las características de la buena práctica de esta actividad traductora y determinar sus elementos interrelacionados. El objetivo general de la revisión consiste en asegurarse de la calidad del producto final de la traducción. No obstante, este objetivo, en nuestra opinión, puede conseguirse sólo a través de un conjunto de objetivos específicos que se complementan entre sí. A continuación trataré de resumir lo que puedo considerar como objetivos específicos de la revisión de traducción:

1-Garantizar que el TL expresa fielmente el sentido del TO.

\footnotetext{
${ }^{7}$ S. Parra Galiano, op. cit., p. 18.
} 
2-Asegurarse de que el TL está adecuado a las características de la lengua de llegada (LL) y a los nuevos destinatarios.

3-Solucionar los problemas de interferencias. Como el revisor no se hunde, igual que el traductor, en los problemas delicados de la traducción, podría poseer una visión de conjunto hacia el TO, algo que le permite liberarse de sus efectos negativos sobre el TL.

4-Eliminar o, al menos, reducir la prevalencia del estilo de redacción propio del traductor sobre el del autor original.

5-Concentrarse en el sentido general del texto, dando más importancia al texto en completo que a sus partes menores. Como el traductor trabaja de una forma fragmentada, centrando su atención en las palabras y frases del texto, la tarea del revisor debe ser la de examinar el texto de manera integral para poder comprobar la adecuación del TL en los distintos aspectos lingüísticos y contextuales.

6-Guardar el equilibrio adecuado entre la traducción libre y la literal, tal y como exige la naturaleza del TO. De esta forma, el revisor puede encontrar soluciones a los dos problemas que podrían amenazar el trabajo del traductor. Según Vázquez Ayora ${ }^{8}$, estos son la ambigüedad causada por la traducción literal, y los cambios de significado causados por la traducción libre. A este respecto, se ha de tener presente que estos problemas se agravan cuanto menor sea la experiencia del traductor.

7-Fomentar el trabajo en equipo y el intercambio de experiencias entre el traductor y el revisor. Las correcciones y mejoras que introduce el revisor le sirven al traductor para ganar nuevas experiencias. De igual modo, la buena traducción le ayuda al revisor a ganar nuevos conocimientos. Por eso, Juncal ${ }^{9}$ insiste en que la revisión es el medio más eficaz, eficiente y económico para enseñar la traducción en el lugar de servicio. Sin embargo, a pesar de la importancia de este objetivo, la naturaleza pragmática del mercado laboral a veces impide lograrlo, ya que no en todos los casos a los proveedores de servicios de traducción les interesa enviar retroalimentaciones a los traductores.

8-Uniformar la terminología utilizada en el TL, además de comprobarse de su buen uso y eliminar las ambigüedades terminológicas.

\footnotetext{
${ }^{8}$ Cfr. G. Vázquez Ayora, Introducción a la traductología, Washington, Georgetown University Press, 1977, p. 394.

${ }^{9}$ J. A. Juncal, "Revisión y estilo de traducción en las Naciones Unidas: ayer y hoy", Panace@ 23, VII (2006), p. 145.
} 
9- Reducir la pereza y el descuido del traductor. En muchos casos, cuando este se entera de que su traducción va a revisarse, trata de mejorar su perfil como traductor profesional, haciendo más esfuerzo en buscar oportunas equivalencias y soluciones a los problemas de traducción que enfrente.

10-Comprobar que el traductor no utiliza un lenguaje neutro, ya que a veces recurre a este medio para escapar de los casos ambiguos o difíciles de traducción, y, por tanto, no asume la responsabilidad de cometer errores.

Son, pues, diez objetivos específicos cuya consecución conduce, a nuestro parecer, a lograr el objetivo general de la revisión de traducción, este es asegurarse de la calidad y corrección de la traducción a dos niveles: la transmisión del sentido del TO, y la adecuación del TL a la cultura de la lengua de llegada.

\section{Fases de la revisión}

La revisión metodológica y sistemática de la traducción exige que el texto traducido pase por varias fases de revisión; no se puede limitar la revisión a una sola etapa. Cuanto mayor sea la relevancia del texto objeto de traducción, el proveedor de servicios de traducción debe cumplir con todas las fases de la revisión. El siguiente gráfico ilustra, a nuestro modo de ver, dichas fases y su secuencia:

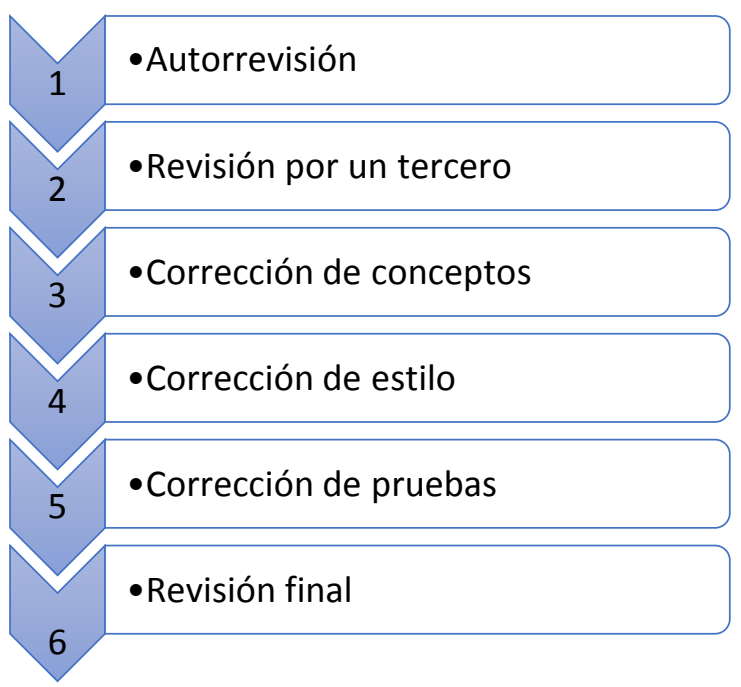

Gráfico 1: Fases de la revisión

Una vez terminada la traducción, el mismo traductor empieza a releer o revisar su propia traducción, algo que podemos denominar como autorrevisión o relectura. Esta es, pues, una fase inherente al proceso de traducción, en la cual el mismo 
traductor del texto verifica el borrador de traducción. Según Mossop, "self-revision [...] is an essential part of translation procedure; skipping it is simply unprofessional" ${ }^{10}$. Sería mucho mejor que el traductor no limitara su revisión a leer el TL sin compararlo con el TO. Asimismo, si dispone de tiempo, sería conveniente dejar la revisión durante un período de tiempo hasta que olvidara sus propias decisiones de traducción, y, por tanto, volver a leer su propia traducción desde la perspectiva de un revisor.

La segunda fase de revisión es aquella realizada por una tercera persona. Aunque esta fase no era antes más que un servicio opcional de valor añadido, durante la última década cobra cada vez más importancia. El revisor es otro traductor con nuevas experiencias y diferentes conocimientos a los del traductor original. Para que su trabajo sea lo más objetivo posible, debe partir de principios y parámetros previamente determinados, aspectos que vamos a estudiar detalladamente en apartados posteriores.

En la tercera fase se corrigen los términos y conceptos por un experto en el tema del texto traducido. La revisión terminológica de conceptos se utiliza mucho en la traducción de textos científico-técnicos. Martínez de Sousa define al corrector de concepto como "una persona especializada que lee un original para corregir errores conceptuales, técnicos o científicos, generalmente derivados de la traducción de una obra" 11 . Este corrector debe verificar la corrección del uso de los términos y su homogeneización en todo el texto, además de asegurarse de la adecuación de los términos a los nuevos destinatarios. No obstante, no tiene derecho a introducir cambios sin consultar al traductor.

En la fase de la corrección de estilo, una persona con los conocimientos necesarios en gramática y ortografía se dedica a corregir los errores morfosintácticos, ortográficos y léxico-semánticos. Esta revisión es necesaria porque el traductor, que durante el proceso de traducción presta más atención al contenido, a veces comete errores formales. Igual que el corrector de concepto, el corrector de estilo debe contactar con el traductor antes de introducir sus cambios.

Luego, el texto se transmite al corrector de pruebas. Es una persona que se ocupa de identificar los pequeños detalles e inadvertencias, centrándose, no en la calidad de la traducción, sino en la calidad lingüística final del producto. Como ejemplos de los errores que deben ser detectados en la fase de la corrección de pruebas señalamos las erratas tipográficas y los elementos que deben ser escritos en

${ }^{10}$ B. Mossop, Revising and Editing for Translators, Manchester, St. Jerome, 2001, p. 91. «La autorrevisión es una parte esencial del proceso de traducción. Eliminarla es simplemente poco profesional» (T. del A.).

${ }^{11}$ J. Martínez de Sousa, Manual de edición y autoedición, Madrid, Ediciones Pirámide, 1999, p. 84. 
letras negritas, cursivas o versalitas. Igual que en las últimas dos fases, el corrector de pruebas tampoco modifica el texto sin consultar al traductor.

Con la revisión final llegamos al último control de calidad al que se somete el texto traducido. En esta fase el revisor añade sus observaciones y comentarios, cualesquiera que sean, convirtiéndose en un receptor del texto traducido; el TL es leído como si fuera escrito originalmente en la LL. Se espera que el texto, llegando a esta fase, carezca de errores fundamentales.

\section{Método de la revisión}

Los especialistas de la traducción no se ponen de acuerdo sobre un determinado método de revisión. Nos referimos con este término a una manera precisa según la cual el revisor debe seguir su trabajo. Algunos autores creen incluso que no se puede señalar con precisión una cierta manera para hacer la revisión, razón por la cual el modo de revisión podría diferenciarse de un revisor a otro, e, incluso, de una revisión a otra, aunque las dos sean llevadas a cabo por el mismo revisor. Entre los expertos que creen en ello se destaca Brian Mossop ${ }^{12}$. Sin embargo, el mismo traductólogo recomienda, si se dispone de tiempo suficiente, que el revisor lea el texto traducido varias veces, de modo que dedica la primera lectura para verificar la corrección del estilo del TL, sacando provecho de no influirse todavía por el TO, ya que aún no lo habrá leído. Seguidamente, en la segunda lectura, el revisor debe centrar su atención en los aspectos relacionados con la precisión de la traducción. El mismo Mossop añade que algunos textos podrían exigir, debido a sus propias características, otras lecturas en las cuales el revisor debe comprobar la corrección de ciertos elementos en el TL, como el formato, los números, las fechas y los nombres, etc.

Al contrario, otros expertos insisten en que, para proceder a la revisión, el revisor debe seguir un método determinado. Dichos autores suelen presentar sus propias propuestas sobre ello. Uno de ellos es Vázquez-Ayora ${ }^{13}$, quien considera que el revisor debe intervenir en cuatro fases:

1) Lectura de la versión o proyecto de traducción en lengua de llegada con el fin de apreciar el efecto total del tema y del estilo.

2) Lectura del texto en lengua original, a grandes rasgos, al objeto de lograr una visión de conjunto y decidir la situación general.

3) Comparación de los textos para verificar la exactitud de las correspondencias y la fidelidad de la traducción en general.

${ }^{12}$ B. Mossop, "Empirical studies of revision: what we know and need to know", The Journal of Specialised Translation 8 (2007), p. 16.

${ }^{13}$ G. Vázquez Ayora, op. cit., p. 39. 
4) Lectura final de la versión en lengua de llegada al objeto de dar los toques finales para lograr una completa cohesión e inteligibilidad y decidir si la comunicación es eficiente y natural.

Como se ve en este método, la revisión debe empezar y terminar por leer el TL, y entre ambas fases viene la lectura del TO y la comparación entre los dos textos, con el propósito de comprobar la corrección de la traducción y añadir las modificaciones y mejoras necesarias.

Horguelin ${ }^{14}$ estima que la revisión debe pasar también por cuatro fases, aunque su orden es diferente:

1) Lectura completa del TO.

2) Lectura comparativa del TO y el TL.

3) Corrección.

4) Lectura del TL una vez revisado.

Se observa en esta propuesta que, a diferencia de la anterior, el autor recomienda al revisor que empiece su trabajo por leer el TO, y esta es la principal divergencia entre ambas propuestas. A este respecto, nosotros creemos que el método sugerido por Vázquez Ayora es más apropiado que el de Horguelin, porque hace que la labor del revisor sea complementaria, no repetitiva, a la del traductor. Si el traductor empieza el proceso de traducción por leer y analizar el TO, sería más conveniente que el revisor comenzara por leer el TL porque este es el momento en que el revisor está mínimamente influido por el TO. La naturaleza de la labor del traductor exige partir siempre del TO, y de las unidades más pequeñas del texto. Por eso, la necesidad al revisor consiste en que este posee una visión más amplia y menos influida por el TO que la del traductor. El revisor debe partir, entonces, de la lectura del TL, y luego se transmite al resto de las fases, como se muestra en el siguiente gráfico, que proponemos como método apropiado de la revisión de traducción:

${ }^{14}$ Cfr. J. I. Perea Sardón, "Revisión asistida por ordenador de traducciones", Universidad de Granada, 2010, pp. 22-23. 


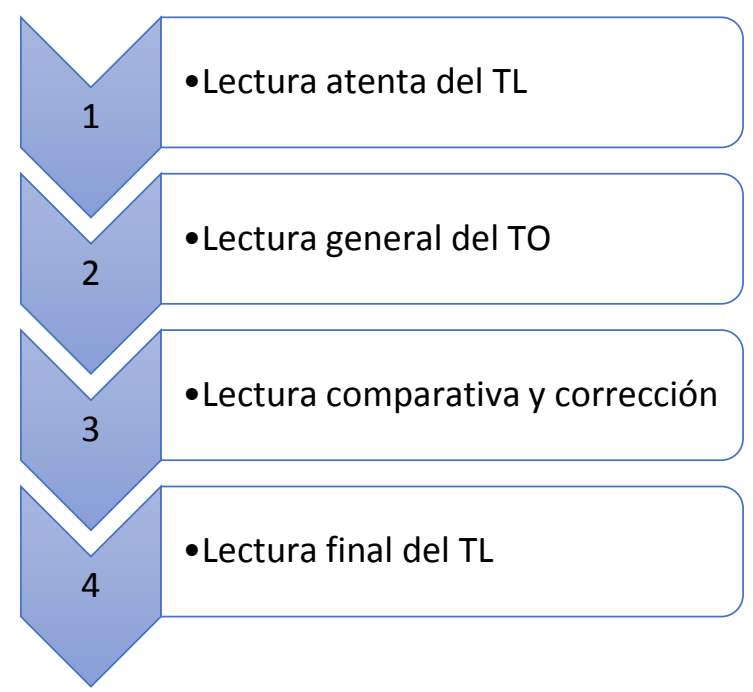

Gráfico 2: Método propuesto de la revisión

\section{Parámetros de la revisión}

La subjetividad inherente a la actividad de la revisión constituye uno de sus problemas más destacados. El revisor que realice su tarea de manera subjetiva tal vez no sea capaz de justificar y argumentar sus correcciones. Al contrario, la actuación objetiva por parte del revisor ayudará a que el traductor acepte sus cambios y modificaciones. A este respecto, Esperanza Tardáguila ${ }^{15}$ considera que la revisión de textos traducidos sólo será aceptable si el que la recibe está convencido de que la revisión efectuada no es el resultado de un juicio intuitivo o subjetivo por parte del revisor. De ahí que el problema de la subjetividad en la revisión necesite como condición previa la existencia de un conjunto de parámetros adecuados.

Los parámetros de revisión responden a esta pregunta: ¿qué hay que verificar o corregir? Son, pues, la guía o puntos de referencia del revisor, que le ayudan a descubrir los errores de traducción que necesitan ser corregidos, ya sean relacionados con el contenido, el lenguaje, la finalidad o la presentación del TL. Parra Galiano ${ }^{16}$ define el parámetro de revisión como "criterio que se utiliza para revisar una traducción y que responde al aspecto o aspectos del TL que se van a verificar para realizar las correcciones y mejoras necesarias". Así, los parámetros tienen por objetivo garantizar la realización de la revisión de una manera objetiva y profesional.

${ }^{15}$ E. Tardáguila, "Reflexiones sobre la revisión de traducciones", Mutatis Mutandis 2, II (2009), p. 372.

${ }^{16}$ S. Parra Galiano, La revisión de traducciones en la traductología ..., op. cit., p. 391. 
Antes de mencionar los parámetros de la revisión, parece oportuno comenzar con señalar los parámetros que conducen el trabajo del traductor. Entre los expertos que establecieron una serie de criterios o parámetros de la traducción se destaca Darbelnet ${ }^{17}$, quien propuso cinco criterios para lograr una buena traducción:

1) La transmisión exacta del mensaje del TO.

2) La consideración de las normas gramaticales vigentes.

3) La elaboración de una traducción idiomática.

4) La conservación del mismo tono que el TO (equivalencia estilística).

5) La plena inteligibilidad del TL (adaptación cultural).

No obstante, muchos traductólogos creen que los criterios de la traducción susodichos no son suficientes ni distintivos de la labor de la revisión. Por eso, aparecieron otras propuestas destinadas precisamente a la actividad de la revisión de traducción. Una de ellas es la de Horguelin y Brunette ${ }^{18}$, en la cual se establecen cinco parámetros para revisar:

1) exactitud (fidelidad al sentido);

2) corrección (respeto del código lingüístico);

3) legibilidad (fácil comprensión);

4) adaptación funcional (tiene en cuenta tono, destinatario); y

5) rentabilidad.

Como se ve, algunos parámetros que figuran en esta propuesta aparecen también en los criterios de traducción propuestos por Darbelnet, además de que el último parámetro, el de la rentabilidad, se encontrará también en los principios de la revisión, aspecto que estudiaremos detalladamente en el apartado siguiente. Esto quiere decir que algunos autores confunden los parámetros con los principios. En nuestra opinión, sería más conveniente considerar la rentabilidad como uno de los principios de la revisión porque es un elemento que ayuda al revisor a tomar la decisión de aceptar la tarea de la revisión, y no a detectar los errores de traducción que necesitan ser corregidos.

La otra propuesta que merece ser mencionada aquí es la de Parra Galiano ${ }^{19}$. Entre las propuestas de establecer parámetros de revisión, creemos que esta es la más completa. La autora española sugiere nueve parámetros, clasificados en cuatro grupos. Antes de detallar dichos parámetros, los resumimos en el gráfico siguiente:

\footnotetext{
${ }^{17}$ Darbelnet, citado por E. Tradáguila, op. cit., p. 372.

${ }^{18}$ Horguelin y Brunette, citado por E. Tradáguila, idem.

${ }^{19}$ S. Parra Galiano, "Propuesta metodológica para la revisión de traducciones: principios generales y parámetros", Trans 11 (2007), p. 204 y ss.
} 


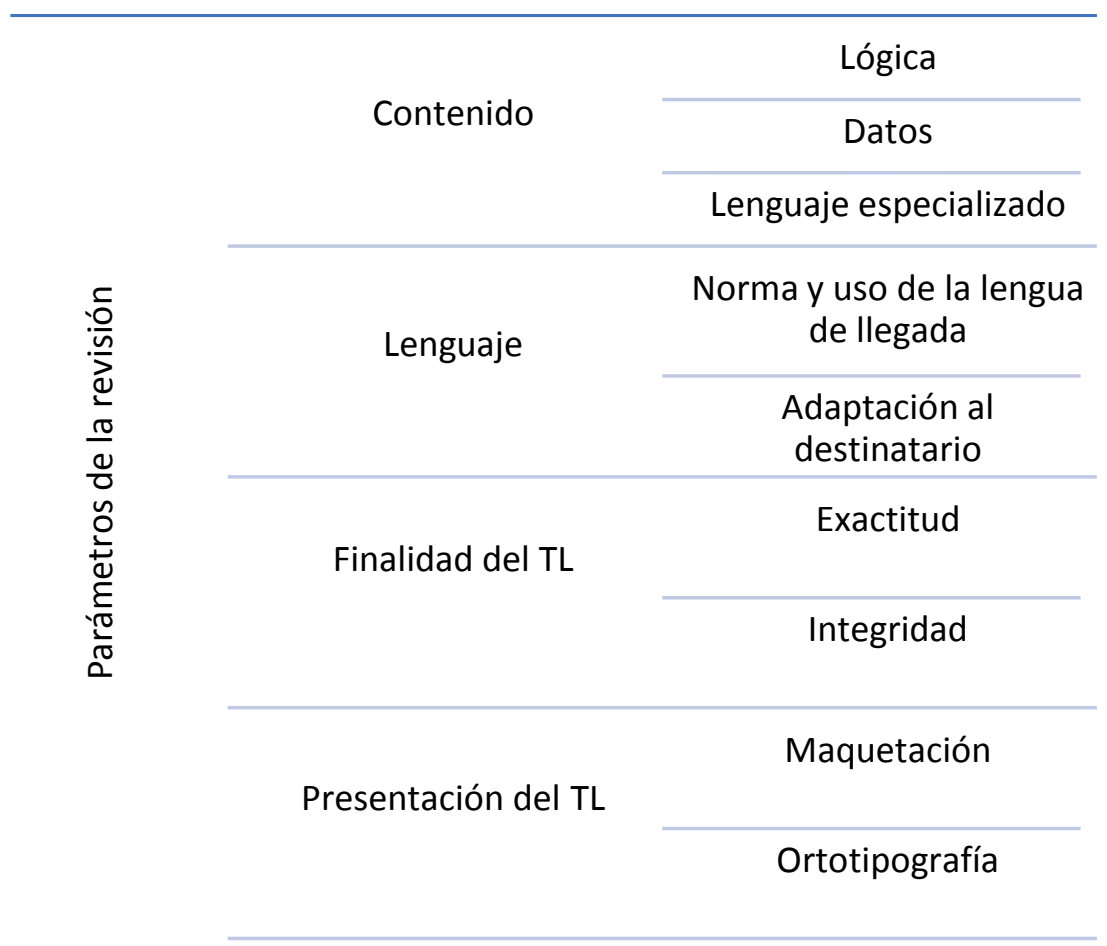

Gráfico 3: Parámetros de la revisión

- Parámetros relativos al contenido:

1) Lógica: verificar si el TL está bien construido, desde el punto de vista formal o lingüístico y desde el punto de vista semántico, algo que depende tanto de la coherencia, como de la cohesión del TL.

2) Datos: identificar la existencia de posibles errores factuales, conceptuales, numéricos o matemáticos en el TL.

3) Lenguaje especializado: considerar la terminología, la fraseología y la estructura del documento, dado que cada tipo de textos tiene sus propios recursos léxicos, sintácticos y retóricos en la lengua de llegada.

- Parámetros relativos al lenguaje:

1) Norma y uso de la lengua de llegada: asegurarse de que el traductor ha respetado el código lingüístico de la lengua de llegada.

2) Adaptación al destinatario: Si el perfil del destinatario del TO no coincide con el del TL, el revisor debe comprobar si el traductor ha modificado el tono mediante un nivel de lengua y registro apropiados.

- Parámetros relativos a la finalidad del TL:

1) Exactitud: garantizar que el sentido del TL es el mismo que el del TO.

2) Integridad: el TL debe transmitir la totalidad del mensaje que contiene el TO, salvo si se trata de una traducción resumen o parcial del TO. 
- Parámetros relativos a la presentación del TL:

1) Maquetación: verificar si la estructura del texto y su disposición global es clara y no induce a confusión. Asegurarse, por ejemplo, de que la numeración relativa a los cuadros, tablas, capítulos, etc., que aparece en el índice corresponde realmente a las páginas de referencia.

2) Ortotipografía: verificar si en el TL se han utilizado de manera apropiada los elementos tipográficos (cursiva, versalita, negrita, subrayado, sangría, espacio, interlineado, etc.) y, si así lo requiere el encargo de traducción, si se han respetado las normas de un determinado libro de estilo.

De lo que antecede se desprende que existen parámetros que cubren la actividad de la revisión en sus distintos niveles. Son criterios que muestran al revisor los elementos que necesitan ser verificados y, si es necesario, corregidos, a nivel del contenido, el lenguaje, la finalidad y la presentación del TL. Si el revisor se da cuenta de todo eso, sabrá cuándo debe introducir cambios y cuándo debe parar; y si introduce cambios, pues tiene lo que justifica sus intervenciones.

\section{Principios de la revisión}

El objetivo principal de establecer principios generales para la revisión de la traducción consiste en la necesidad de saber las normas en las cuales se basa esta práctica, la forma de dirigirla y las consideraciones de las cuales debe partir el revisor para determinar el tipo de los cambios que puede introducir y los límites de sus intervenciones. De ahí, los principios de la revisión tratan de contestar a la pregunta de cómo se puede dirigir la actividad de la revisión.

La revisión no significa de ningún modo que se dé carta blanca al revisor, modificando el TL como quiera; cuanto menos sean las intervenciones del revisor, no se crearán nuevos errores durante la fase de la revisión. A este respecto, merece mencionarse el estudio de Alexander Künzli ${ }^{20}$, en el cual, tras un estudio de la revisión realizada por diez traductores profesionales de un texto legal, concluye que en cuatro de los diez casos estudiados el resultado final de la revisión era de peor calidad que el borrador inicial. Concluimos de este estudio que hay una alta probabilidad, estimada en 40\%, de que la revisión deforme, y no mejore, el TL. La razón de ello radica, sin duda, en los cambios innecesarios que a veces introduce el revisor, y la confusión que aflige al traductor a la hora de insertar estas "modificaciones" en el TL.

Un estudio como este no quiere decir, naturalmente, que el proceso de la revisión sea inútil, sino que la función del revisor debe limitarse a la corrección de los errores, por un lado, y, por otro, no introducir cambios innecesarios en el TL.

${ }^{20}$ A. Künzli, citado por J. I. Perea Sardón, op. cit., p. 20. 
En nuestra opinión, los cambios de este tipo no solamente empeoran el TL, sino también desperdician el tiempo. De ahí destaca la importancia de limitar la tarea del revisor a corregir errores que no pueden ignorarse, o renunciar la revisión de las traducciones repletas de errores.

A continuación, explicaremos los principios de la revisión de los cuales debe partir el revisor para delimitar sus intervenciones $\mathrm{y}$, por tanto, dirigir bien el proceso de la revisión. A nuestro juicio, dichos principios pueden clasificarse en tres grupos principales, tal como muestra el siguiente gráfico:

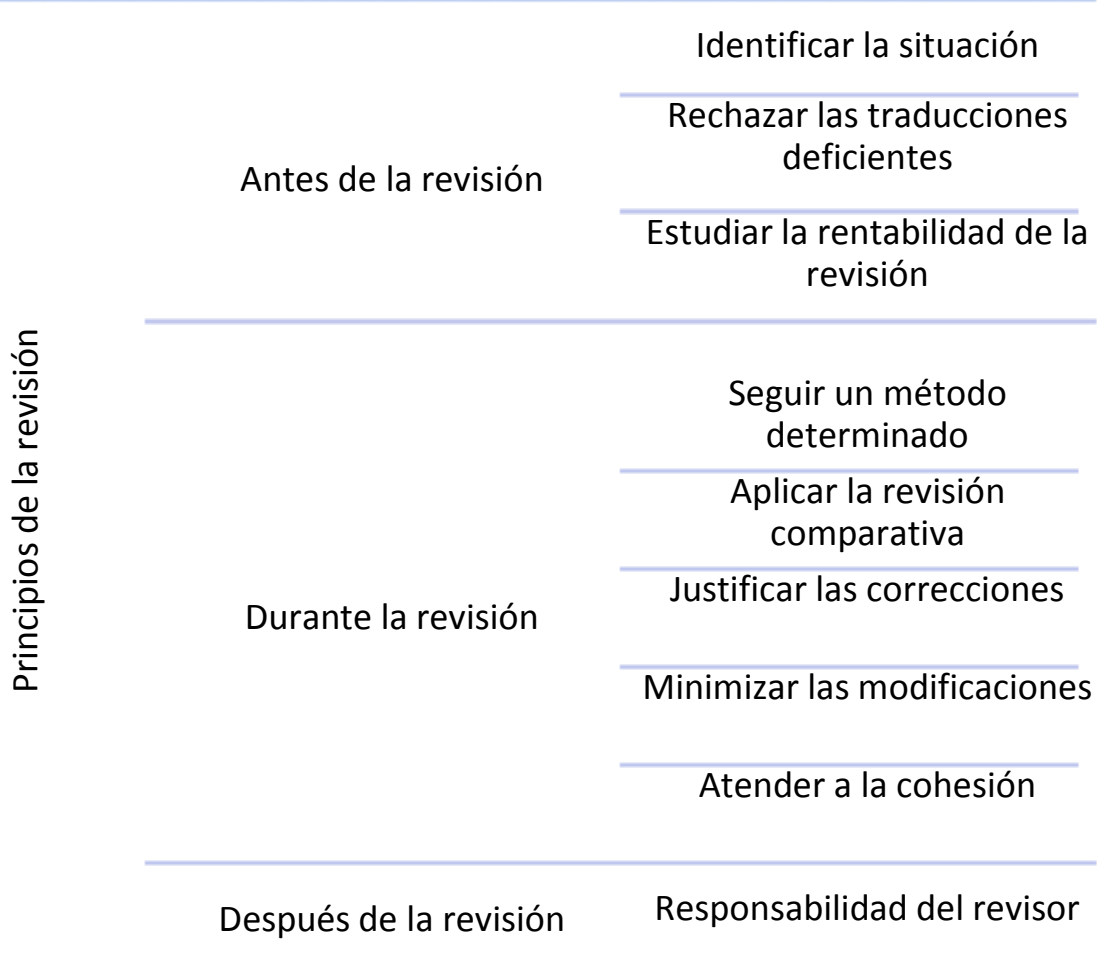

Gráfico 4: Principios de la revisión

- Principios a seguir por parte del revisor antes de empezar el proceso de la revisión:

1) Identificar la situación: determinar el tipo, la finalidad y el destinatario del texto que se va a verificar, y el tiempo que puede dedicar a la revisión.

2) Rechazar toda traducción que considere muy deficiente. Una traducción defectuosa exige ser reescrita por parte del revisor, y esta no es su función.

3) Estudiar la rentabilidad de la revisión. El revisor tiene derecho a rechazar la revisión si no es rentable. 
- Principios a seguir por parte del revisor durante el proceso de la revisión:

1) Seguir un método determinado. En un apartado anterior hemos dejado constancia de que el método más apropiado en nuestra opinión debe empezar por la lectura atenta del TL, pasando por la lectura del TO, y la lectura comparativa y corrección, y llegando últimamente a una lectura final del TL.

2) Aplicar la revisión comparativa. El TL debe ser revisado en comparación al TO.

3) Justificar las correcciones y modificaciones. El revisor debe estar dispuesto a justificar y argumentar cada corrección utilizada en el TL, sobre todo, aquellas que necesiten una prueba.

4) Minimizar las modificaciones cuanto se pueda. El revisor no debe convertir sus propias preferencias en reglas que deben ser seguidas.

5) Atender a la cohesión. Si el TL ha sido traducido por varias personas, el revisor deberá ocuparse de homogeneizar el estilo, la terminología utilizada y la presentación física del mismo.

- Principios a seguir por parte del revisor después del proceso de la revisión:

1) Responsabilidad del revisor: En aquellos casos en los que el traductor no tenga la posibilidad de ver el TL una vez revisado, para conocer las correcciones y mejoras realizadas y argumentar sus soluciones, el responsable del TL definitivo es el revisor.

En síntesis, los principios de la revisión son la guía o puntos de referencia del revisor, que tienen por objetivo garantizar la realización de la revisión de una manera objetiva y profesional. 


\section{Conclusiones}

La revisión de la traducción es uno de los medios más importantes de garantizar la calidad de la traducción. No obstante, este medio no será bastante eficiente ni dará su fruto deseado hasta que se practique de una forma objetiva y metodológica, basada en parámetros y principios establecidos previamente.

El presente estudio ha pretendido ayudar a esbozar la práctica objetiva de la revisión, mostrando que hay varios objetivos que deben ser perseguidos por el revisor. Un revisor que no se da cuenta de los objetivos de la revisión, le resultará difícil percibir la realidad de su profesión ni su finalidad.

Hemos dejado constancia, asimismo, de que la revisión debe pasar por seis fases y que no se puede reducirla a una sola. Cada fase complementa a la que le precede hasta que salga el TL en la mejor forma posible.

Por otro lado, se ha puesto de manifiesto que el revisor debe adoptar un método claro y determinado. Hemos propuesto un método que empieza por la lectura atenta del TL, pasando por la lectura general del TO, luego la lectura comparativa y corrección, y terminando con una lectura final del TL.

Posteriormente, hemos demostrado que existe una serie de parámetros que conducen el trabajo del revisor y que tienen por objetivo descubrir los errores de traducción que necesitan ser corregidos. Del mismo modo, hemos aclarado que la revisión cuenta con un conjunto de principios de los cuales debe partir el revisor para concretar sus intervenciones y saber sus límites.

Estos son, pues, cinco aspectos que, en nuestra opinión, forman juntos la práctica objetiva y metodológica de la tarea de la revisión. Establecer objetivos, fases, método, parámetros y principios de la revisión de la traducción hace que todas las partes involucradas en el proceso de la traducción (traductor, cliente, proveedor de servicios de traducción, etc.) se aseguren de que el revisor no parte en su tarea de consideraciones subjetivas o preferencias personales, sino que se basa en normas metodológicas claras y principios objetivos fijos. 


\section{Bibliografía}

AENOR. Norma Española UNE-EN 15038. Servicios de traducción. Requisitos para la prestación del servicio. Madrid: AENOR, 2006.

Arthern, Peter. "Four eyes are better than two". Translating and the Computer 8: A Profession on the Move. Picken, C. (ed.). London: Aslib. 1987: 14-26.

Comisión Europea, Dirección General de Traducción, Departamento de Lengua Española. Manual de revisión. Bruselas y Luxemburgo: Comisión Europea, 2010.

Juncal, Julio Ángel. "Revisión y estilo de traducción en las Naciones Unidas: ayer y hoy".Panace@ 23, VII (2006): 145-148.

Martínez de Sousa, José. Manual de edición y autoedición. Madrid: Ediciones Pirámide, 1999.

Mossop, Brian. Revising and Editing for Translators. Manchester: St. Jerome, 2001.

- "Empirical studies of revision: what we know and need to know". The Journal of Specialised Translation 8 (2007): 5-20.

Parra Galiano, Silvia. "La revisión de traducciones en la traductología: aproximación a la práctica de la revisión en el ámbito profesional mediante el estudio de casos y propuestas de investigación". Universidad de Granada, 2005.

- "Propuesta metodológica para la revisión de traducciones: principios generales y parámetros". Trans 11 (2007): 197-214.

Perea Sardón, José Ignacio. "Revisión asistida por ordenador de traducciones". Universidad de Granada, 2010.

Real Academia Española (ed.). Diccionario de la Lengua Española. Madrid: Espasa Calpe, 2014, 23 a ed.

Tardáguila, Esperanza. "Reflexiones sobre la revisión de traducciones". Mutatis Mutandis 2, II (2009): 367-376.

Valverde, Íñigo. "Algunas consideraciones sobre la revisión". Puntoycoma (Boletín de los traductores españolas de las instituciones de la Unión Europea) 117 (2010): 34-38.

Vázquez Ayora, Gerardo. Introducción a la traductología. Washington: Georgetown University Press, 1977. 